\title{
Applications of the Nambu-Jona-Lasinio model to the partonic structure of the pion ${ }^{\star \star \star \star}$
}

\author{
Wojciech Broniowski ${ }^{1,2, a}$, and Enrique Ruiz Arriola ${ }^{3, b}$ \\ ${ }^{1}$ Institute of Physics, Jan Kochanowski University, 25-406 Kielce, Poland \\ ${ }^{2}$ Institute of Nuclear Physics PAN, 31-342 Cracow, Poland \\ ${ }^{3}$ Departamento de Física Atómica, Molecular y Nuclear and Instituto Carlos I de \\ Física Teórica y Computacional, Universidad de Granada, 18071 Granada, Spain
}

Received 22 March 2020 / Accepted 27 October 2020

Published online 21 December 2020

\begin{abstract}
We present a brief review of results of chiral quark models for soft matrix elements in the pion state, appearing in high-energy processes as well as accessible in present and future lattice studies. A particular attention is paid to the recently explored double parton distribution functions of the pion.
\end{abstract}

\section{Introduction}

David has been successfully using quark models all over his career, including applications to dense matter where the otherwise usually confined quarks may be de-confined and give rise to stars with a quark core $[1,2]$. In this talk we focus on other aspects of chiral quark models, such as the Nambu-Jona-Lasinio (NJL) model, applied to the domain where they were originally designed for, namely the soft limit in the vacuum. There the quarks remain confined, but the chiral symmetry is broken, leading to rich dynamical predictions. It is not so commonly known that in this case the model explains numerous features of the pion (in general, the Goldstone bosons), both in low- and high-energy processes, whenever the soft-hard factorization holds (see [3] for a detailed review). These results, amended with the necessary QCD evolution which generates radiatively the gluon degrees of freedom, compares very favorably to the available experimental and lattice data. We briefly review some of these results and then pass to a recent topic of double parton distribution functions ( $\mathrm{dPDF}$ ) in the pion [4-6] evaluated in chiral quark models followed with the DGLAP evolution. We discuss the issue of partonic correlations and the proposed measures based on the Mellin moments of dPDFs [6].

\footnotetext{
* Dedicated to David Blaschke on the occasion of his 60th birthday. Presented by WB at The 40th Max Born Symposium Three Days on Strong Correlations in Dense Matter, 9-12 October 2019, Wrocław, Poland.

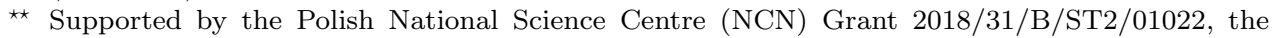
Spanish Ministerio de Economia y Competitividad and European FEDER funds Grant FIS201785053-C2-1-P, and Junta de Andaluca Grant FQM-225.

a e-mail: Wojciech.Broniowski@ifj.edu.pl

b e-mail: earriola@ugr.es
} 


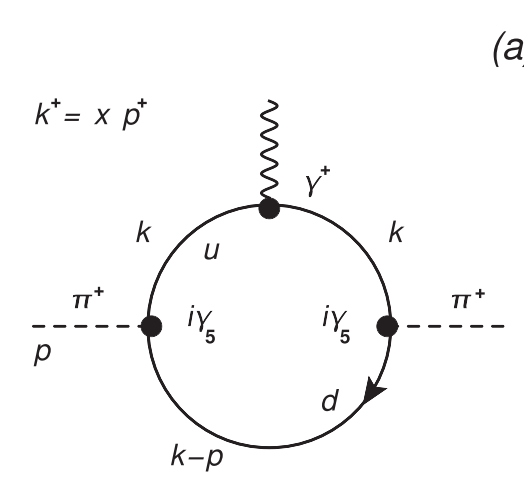

(a)

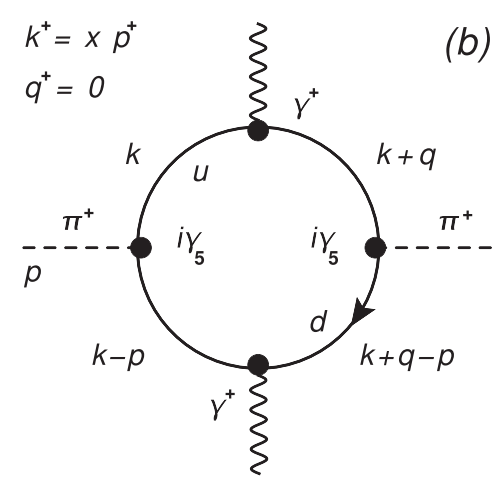

Fig. 1. Diagrams to evaluate the single (a) and double (b) valence quark distributions of $\pi^{+}$at the leading- $N_{c}$ order in the NJL model. In diagram (b) integration over $q^{-}$is carried out.

\section{Basic formalism}

The field-theoretic definition of the single parton distribution function (sPDF) involves a diagonal matrix element of bilinear operators in a hadronic state (see [7] and references therein), namely

$$
D_{j}(x)=\left.\int \frac{d z^{-}}{2 \pi} e^{i x z^{-} p^{+}}\left\langle p\left|\mathcal{O}_{j}(0, z)\right| p\right\rangle\right|_{z^{+}=0, \boldsymbol{z}=\mathbf{0}}
$$

Here $p$ is the momentum of the hadron, $x$ is the Bjorken variable interpreted as the fraction of the light-cone momentum of the hadron carried out by the struck parton, and $\mathcal{O}_{j}(0, z)$ is a bilocal operator which for the quarks and anti-quarks considered here and in the applied light-cone gauge takes the form

$$
\begin{aligned}
& \mathcal{O}_{q}(y, z)=\frac{1}{2} \bar{q}\left(y-\frac{z}{2}\right) \gamma^{+} q\left(y+\frac{z}{2}\right), \\
& \mathcal{O}_{\bar{q}}(y, z)=-\frac{1}{2} \bar{q}\left(y+\frac{z}{2}\right) \gamma^{+} q\left(y-\frac{z}{2}\right) .
\end{aligned}
$$

The light-cone coordinates are introduced as $v^{ \pm}=\left(v^{0} \pm v^{3}\right) / \sqrt{2}$, whereas the boldface indicates the transverse components, $\boldsymbol{v}=\left(v^{1}, v^{2}\right)$. The quark-pion coupling is pointlike, as follows from the NJL model.

For the double parton distribution functions (dPDF) one has, analogously, a matrix element involving two bilocal currents [7],

$$
\begin{array}{r}
D_{j_{1} j_{2}}\left(x_{1}, x_{2}, \boldsymbol{b}\right)=2 p^{+} \int d y^{-} \frac{d z_{1}^{-}}{2 \pi} \frac{d z_{2}^{-}}{2 \pi} e^{i\left(x_{1} z_{1}^{-}+x_{2} z_{2}^{-}\right) p^{+}} \\
\quad \times\left.\left\langle p\left|\mathcal{O}_{j_{1}}\left(y, z_{1}\right) \mathcal{O}_{j_{2}}\left(0, z_{2}\right)\right| p\right\rangle\right|_{z_{1}^{+}=z_{2}^{+}=y^{+}=0, \boldsymbol{z}_{1}=\boldsymbol{z}_{2}=\mathbf{0}}
\end{array}
$$

where indices 1,2 refer to the two partons. Note that there is an extra argument of this object, namely, the relative transverse distance between the partons, $\boldsymbol{b}$.

The simple meaning of the above definitions in the momentum space is illustrated in Figure 1, where for definiteness we take the case of the charged pion and use the large- $N_{c}$ limit, which amounts to evaluating the one quark loop. We note that for $\mathrm{dPDF}$ of Figure $1 \mathrm{~b}$ there is a momentum flow between the two probing operators. Integration over $q^{-}$imposes the constraint $y^{+}=0$ from equation (3), whereas the 


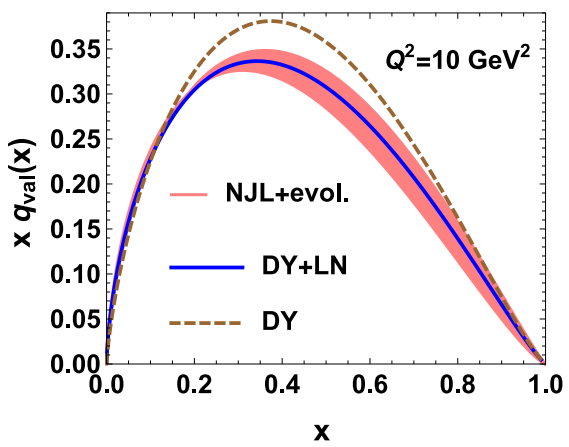

Fig. 2. Comparison of the NJL mode followed with LO DGLAP evolution (band) to the extraction of the valence pion sPDF made in [9]. The dashed line uses the $\pi A$ Drell-Yan data, and the solid line combines them with the HERA leading-neutron electro-production data.

transverse component $\mathbf{q}$ is the Fourier-conjugate variable corresponding to $\mathbf{b}$. The evaluation leads to simple results presented in the sections below.

As discussed extensively in [3], the evaluation according to the diagrams of Figure 1 corresponds to the quark model scale $\mu_{0}$, where no gluons are present and the constituent quark and anti-quark saturate the momentum sum rule, $\langle x\rangle_{\mu_{0}}=1$. Fits to phenomenological sPDF provide the value $\mu_{0} \sim 320 \mathrm{MeV}$ (see, e.g., [8]). The matching condition between the model and QCD for an observable $A$ is imposed by the condition

$$
\left.A\left(x, \mu_{0}\right)\right|_{\text {model }}=\left.A\left(x, \mu_{0}\right)\right|_{\mathrm{QCD}} .
$$

Then, the QCD evolution is carried out to higher scales $\mu$, where comparison to the experimental data of lattice simulations is possible. This process, here performed at the leading order, generates radiatively the gluons. The QCD evolution is a crucial ingredient of the approach.

\section{Single parton distributions of the pion}

Let us first recall the results for sPDF of the pion, first obtained by Davidson and one of us (ERA) [10] by analyzing the forward Compton scattering amplitude in the Bjorken limit. ${ }^{1}$ There, at the quark model scale and in the strict chiral limit of $m_{\pi}=0$, one gets (notation for $\pi^{+}$, other pion states are obtained via the isospin symmetry)

$$
q_{\mathrm{val}}(x) \equiv D_{u}(x)=D_{\bar{d}}(x)=1 \times \theta(x) \theta(1-x)
$$

We note the proper support, normalization, and a uniform distribution in the ${ }^{+}$ momentum fraction $x$.

When evolved to higher scales, the results agree very well with the phenomenological extractions of $q_{\mathrm{val}}(x)$ from the data. The comparison to the Fermilab E615 Drell-Yan data can be found, e.g., in [8]), so here in Figure 2 we confront the

\footnotetext{
${ }^{1}$ One technical aspect of high relevance in the calculation is the choice of the NJL regularization, which needs to preserve gauge invariance and chiral symmetry, as well as their corresponding WardTakahashi identities.
} 
model to a recent phenomenological extraction of [9], corresponding to the scale $\mu^{2}=Q^{2}=10 \mathrm{GeV}^{2}$. We note a very good agreement of the model (the band reflects the uncertainty in the initial scale, $\mu_{0}=313_{-10}^{+20} \mathrm{MeV}[8]$ ) to the analysis of [9]. In particular, we note an excellent agreement with the analysis combining the $\pi A$ Drell-Yan data and the HERA leading-neutron electro-production data. A very recent analysis [11] confirms this agreement when we take $\mu_{0}=374 \mathrm{GeV}$ to get 0.55 momentum fraction of the valence quarks at $Q^{2}=5 \mathrm{GeV}^{2}$.

Further, we wish to comment of the behavior of $q_{\mathrm{val}}(x)$ at $x \rightarrow 1$, which recently has been a subject of heated discussion. With the applied DGLAP evolution from $Q_{0}=313 \mathrm{MeV}$, this behavior is given by [8]

$$
q_{\mathrm{val}}(x) \sim(1-x)^{4 C_{f} / \beta_{0} \log \left[\alpha\left(Q_{0}\right) / \alpha(Q)\right]}=(1-x)^{1.24} .
$$

The power clearly evolves with the scale $Q$. We stress a very good agreement of this behavior in our model with the phenomenological extraction, cf. Figure 2.

Admittedly, our analysis as well as the quoted experimental extraction [9] do not account for the soft-gluon resummation effects, which flatten the curve near the thereshold, $x \rightarrow 1$ [12]. In comparisons, such effects should be included both at the theoretical and the experimental side.

There are numerous other results for the pion that can be computed with this scheme (chiral quark model followed with QCD evolution), all of them giving a fair comparison, whenever available, with the experimental or lattice data. Notable examples are the parton distribution amplitude (PDA) [13], the generalized parton distribution functions (GPD) [8], or the quasi parton distributions [14,15].

\section{Double parton distributions of the pion}

Now we pass to our main topic of this talk, namely, the predictions for the valence $\mathrm{dPDF}$ of the pion. The evaluation of the diagram from Figure $1 \mathrm{~b}$ yields (in the chiral limit of $\left.m_{\pi}=0\right)$ the result $[4-6]$

$$
D_{u \bar{d}}\left(x_{1}, x_{2}, \boldsymbol{q}\right)=1 \times \delta\left(1-x_{1}-x_{2}\right) \Theta F(\mathbf{q}),
$$

where the $\delta$ function reflects the conservation of the ${ }^{+}$components of the momentum and the $\Theta$ indicates the proper support $0 \leq x_{1}, x_{2} \leq 1$. The form factor $F(\mathbf{q})$ depends on the adopted regularization scheme, which is necessary to remove the hard momentum contribution from the model. Analogously to the case of sPDF, the distribution in $x_{1}$ or $x_{2}$ is uniform. The factorization of the longitudinal and transverse dynamics holds in the strict chiral limit.

First, let us bring up a very general formal aspect of dPDFs, namely, the GauntStirling (GS) sum rules [16]. These identities hold for the special case of $\mathbf{q}=0$,

$$
D_{i j}\left(x_{1}, x_{2}, \mathbf{q}=0\right) \equiv D_{i j}\left(x_{1}, x_{2}\right),
$$

and link the marginal projections/moments of dPDFs to sPDFs in a way typical of probability distributions. They follow from a decomposition of the parton operators in a basis of the light-front wave functions [17]

$$
\sum_{i} \int_{0}^{1-x_{2}} d x_{1} x_{1} D_{i j}\left(x_{1}, x_{2}\right)=\left(1-x_{2}\right) D_{j}\left(x_{2}\right),
$$



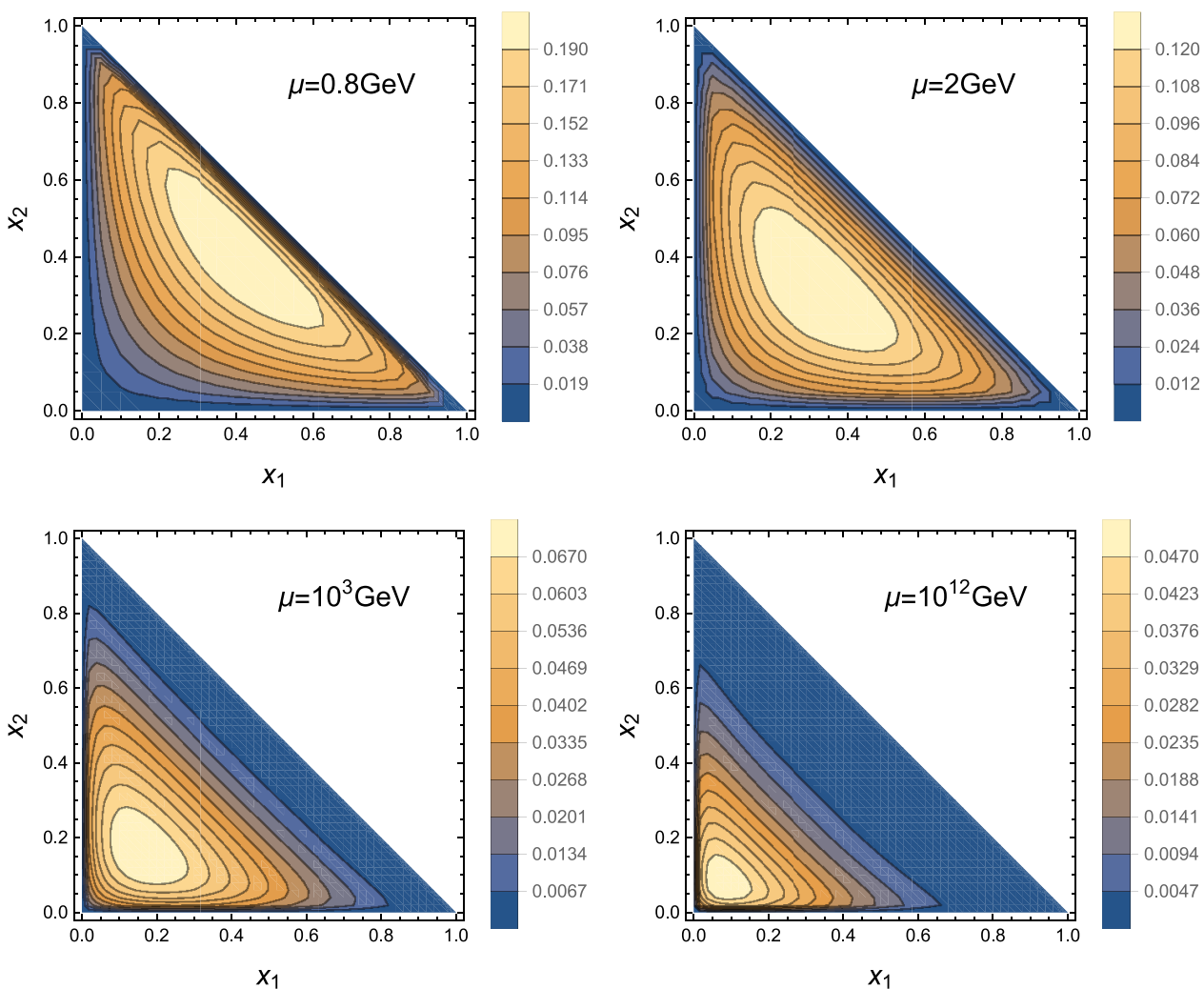

Fig. 3. Valence dPDF of the pion, $x_{1} x_{2} D_{\mathrm{ud}}\left(x_{1}, x_{2}\right)$, evolved with the DGLAP equations to subsequent scales $\mu$ indicated in the panels. The initial condition at the quark model scale $\mu_{0}$ is the singular distribution of equation (7) (taken from [6]).

$$
\int_{0}^{1-x_{2}} d x_{1} D_{i_{\mathrm{val}} j}\left(x_{1}, x_{2}\right)=\left(N_{i_{\mathrm{val}}}-\delta_{i j}+\delta_{\overline{i j}}\right) D_{j}\left(x_{2}\right),
$$

where $i_{\text {val }}$ is the difference of the parton $(i)$ and anti-parton $(\bar{i})$ distributions, with $N_{i_{\mathrm{val}}}=\int_{0}^{1} d x D_{i_{\mathrm{val}}}(x)$. In numerous approaches on the market, the GS sum rules are non-trivial to satisfy $[16,18-20]$. We have shown in $[21,22]$ that a successful approach follows from a top-down method, starting from an $n$-parton distributions with $\delta\left(1-x_{1}-\ldots-x_{n}\right)$ from momentum conservation, which yields the distributions with lower number of partons via subsequent marginal projections.

We note that the NJL form of equation (7) explicitly satisfies the GS sum rules, as the model obeys all constraints from the Lorentz covariance and symmetries. Furthermore, as the sum rules hold when the QCD evolution is applied (see below), the feature holds at any scale.

The scheme to evolve dPDFs has been derived long ago [23,24]. An efficient method is based on the Mellin moments, similarly to the case of sPDFs. Details are presented in $[6,21]$.

Our results for the valence $\mathrm{dPDF}$ of the pion (conventionally multiplied with $x_{1} x_{2}$ ), evolved to increasing scales $\mu$, are shown in Figure 3 . We note that, as expected from the DGLAP evolution, the strength shifts to lower $x_{1}$ and $x_{2}$ as $\mu$ increases. Note that the initial condition for the evolution is distributed singularly along the $x_{1}=x_{2}$ line, as given in equation (7). The evolution washes out this behavior, filling 

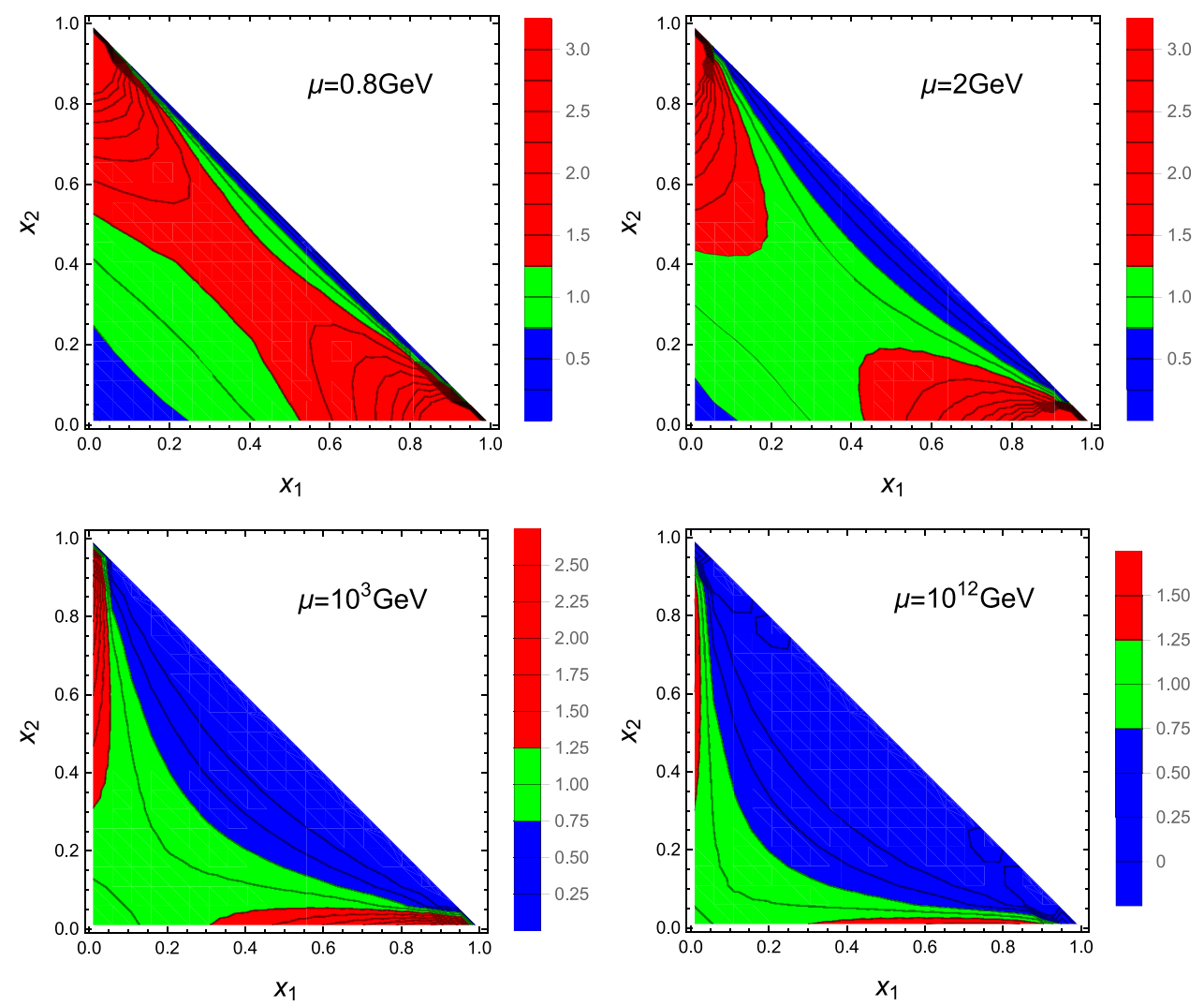

Fig. 4. Correlation $D_{\mathrm{u} \overline{\mathrm{d}}}\left(x_{1}, x_{2}\right) / D_{u}\left(x_{1}\right) D_{\bar{d}}\left(x_{2}\right)$ at various evolution scales $\mu$ indicated in the panels. The regions marked in green have the correlation within $20 \%$ of unity. (taken from $[6])$.

all the triangle with $x_{1}+x_{2} \leq 1$. Of course, as the evolution generates radiatively more partons, the two valence partons do not need to carry all the momentum, hence $x_{1}+x_{2}$ can be smaller than 1 .

Next, in Figure 4 we show our results for the correlation $D_{\mathrm{ud}}\left(x_{1}, x_{2}\right) / D_{u}\left(x_{1}\right) D_{\bar{d}}\left(x_{2}\right)$. This is an important measure, as whenever it departs significantly from 1 , one cannot use the factorization assumption frequently made in analyses of the double parton scattering (see [25] for a recent review). The regions in the plots marked with green have the correlation within $25 \%$ from the unity, indicated the region where factorization approximately works, whereas outside of this region it is significantly broken. We note that at low $x_{1}$ and $x_{2}$ increasing the evolution scale brings the correlation ratio closer to 1 , which is in line with the conclusions of [26] for the gluon distributions in the nucleon.

As proposed in [6], a very practical measures of correlation which hopefully could be probed in the upcoming lattice analyses, are based on the ratios of the Mellin moments for the valence distributions,

$$
\frac{\left\langle x_{1}^{n} x_{2}^{m}\right\rangle}{\left\langle x_{1}^{n}\right\rangle\left\langle x_{2}^{m}\right\rangle} .
$$

The key point is that these ratios are independent of the evolution scale, since the corresponding factors involving the anomalous dimensions cancel out. This feature 
Table 1. The ratios of the double to single valence moments, $\left\langle x_{1}^{n} x_{2}^{m}\right\rangle /\left\langle x_{1}^{n}\right\rangle\left\langle x_{2}^{m}\right\rangle$, in the NJL model, equation (11). Rows and columns correspond to $n$ and $m$. These ratios are independent of the evolution scale $\mu$.

\begin{tabular}{lllll}
\hline & 1 & 2 & 3 & 4 \\
\hline 1 & $2 / 3$ & $1 / 2$ & $2 / 5$ & $1 / 3$ \\
2 & $1 / 2$ & $3 / 10$ & $1 / 5$ & $1 / 7$ \\
3 & $2 / 5$ & $1 / 5$ & $4 / 35$ & $1 / 14$ \\
4 & $1 / 3$ & $1 / 7$ & $1 / 14$ & $5 / 126$ \\
\hline
\end{tabular}

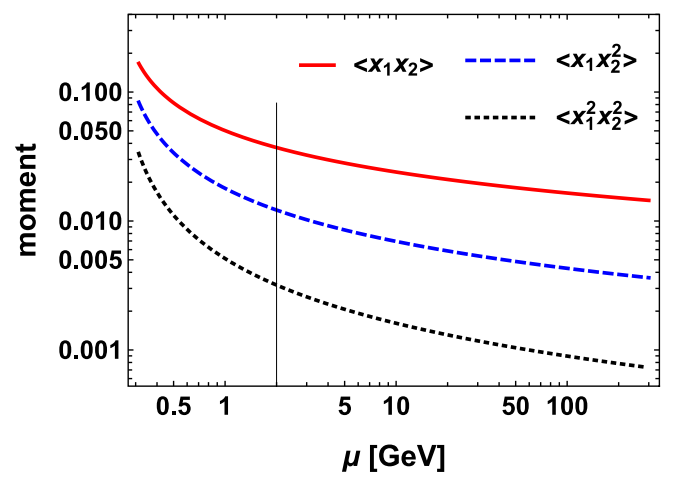

Fig. 5. Lowest Mellin moments of the valence dPDF of the pion, plotted as functions of the evolution scale. The vertical line indicates the typical lattice scale of $2 \mathrm{GeV}$.

occurs only for the valence case, where the evolution does not mix dDPFs and sPDFs via the so called inhomogeneous term. In the NJL model, we have the simple formula

$$
\frac{\left\langle x_{1}^{n} x_{2}^{m}\right\rangle}{\left\langle x_{1}^{n}\right\rangle\left\langle x_{2}^{m}\right\rangle}=\frac{(1+n) !(1+m) !}{(1+n+m) !} .
$$

The lowest values of these ratios are listed in Table 1 , and the evolution of a few lowest moments is presented in Figure 5.

Finally, we discuss the transverse form factor $F(\mathbf{q})$, appearing in equation (7). Its form depends on the regularization scheme of the NJL model, and for fundamental reasons can only be trusted for soft external momenta. In the Spectral Quark Model $(\mathrm{SQM})[27]$ it has a very simple form

$$
F(\boldsymbol{q})=\frac{m_{\rho}^{4}-\boldsymbol{q}^{2} m_{\rho}^{2}}{\left(m_{\rho}^{2}+\boldsymbol{q}^{2}\right)^{2}} .
$$

In the NJL model with the Pauli-Villars (PV) subtraction a numerically very similar result follows, as can be seen from Figure 6 . The region with $|\boldsymbol{q}|>1 \mathrm{GeV}$, where the form factor goes negative, is outside of the validity of the model.

The corresponding effective cross section for the double parton scattering coincides (in SQM) with the geometric cross section, namely

$$
\sigma_{\mathrm{eff}}=\frac{1}{\int \frac{d^{2} \boldsymbol{q}_{\perp}}{(2 \pi)^{2}} F\left(\boldsymbol{q}_{\perp}\right) F\left(-\boldsymbol{q}_{\perp}\right)}=\pi \frac{12}{m_{\rho}^{2}}=\pi\left\langle b^{2}\right\rangle=23 \mathrm{mb} .
$$




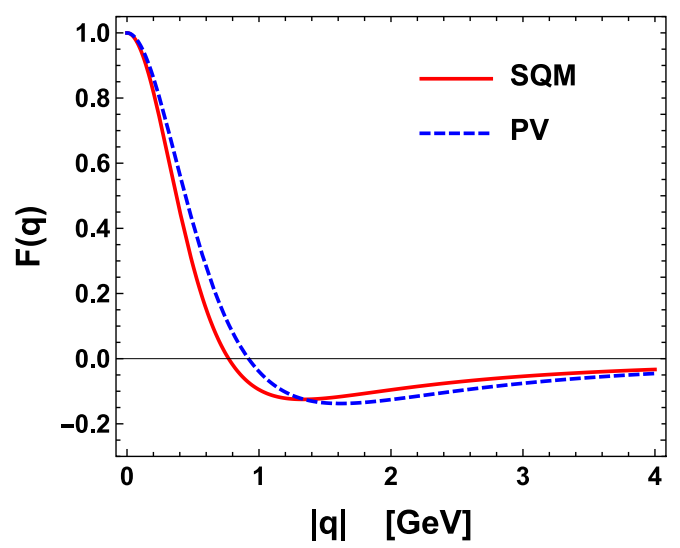

Fig. 6. Valence dPDF form factor of the pion in the SQM (solid line) and in NJL with PV regularization (dashed line), plotted as a function of the transverse momentum.

\section{Summary}

To summarize, here are our main points:

- The topic of the double parton correlations is driven by recent experimental evidence as well as by possible future lattice studies.

- Our results are obtained in the NJL model which is a simple field theory of the pion in the soft regime based on the spontaneous chiral symmetry breaking. It is a covariant calculation, with all symmetries preserved, which leads to proper formal features. In particular, the GS sum rules are satisfied. The QCD evolution to higher scales is a crucial ingredient of the approach.

- The model leads, in the chiral limit, to the longitudinal-transverse factorization, whereas there is no factorization in the $x_{1}$ and $x_{2}$ variables, which are correlated due to the momentum conservation.

- The correlation ratio at low $x_{1}$ and $x_{2}$ is brought close to 1 with increasing evolution scale.

- The appropriate ratios of the Mellin moments do not depend on the evolution scale, hence are particularly convenient. They could be probed in future lattice simulations.

\section{Author contribution statement}

Both authors have contributed equally to all stages of the research and preparation of this article.

Open Access This is an open access article distributed under the terms of the Creative Commons Attribution License (https://creativecommons.org/licenses/by/4.0), which permits unrestricted use, distribution, and reproduction in any medium, provided the original work is properly cited. 
Publisher's Note The EPJ Publishers remain neutral with regard to jurisdictional claims in published maps and institutional affiliations.

\section{References}

1. M. Alford, D. Blaschke, A. Drago, T. Klahn, G. Pagliara, J. Schaffner-Bielich, Nature 445, E7 (2007)

2. M. Shahrbaf, D. Blaschke, A.G. Grunfeld, H.R. Moshfegh, Phys. Rev. C 101, 025807 (2020)

3. E. Ruiz Arriola, Acta Phys. Pol. B 33, 4443 (2002)

4. W. Broniowski, E. Ruiz Arriola, PoS LC2019, 31 (2019)

5. A. Courtoy, S. Noguera, S. Scopetta, JHEP 12, 045 (2019)

6. W. Broniowski, E. Ruiz Arriola, Double Parton Distribution of Valence Quarks in the Pion in Chiral Quark Models, arXiv:1910.03707

7. M. Diehl, PoS DIS 2010, 223 (2010)

8. W. Broniowski, E. Ruiz Arriola, K. Golec-Biernat, Phys. Rev. D 77, 034023 (2008)

9. P.C. Barry, N. Sato, W. Melnitchouk, C.-R. Ji, Phys. Rev. Lett. 121, 152001 (2018)

10. R. Davidson, E. Ruiz Arriola, Phys. Lett. B 348, 163 (1995)

11. I. Novikov et al., Parton Distribution Functions of the Charged Pion Within The xFitter Framework, arXiv:2002.02902

12. M. Aicher, A. Schafer, W. Vogelsang, Phys. Rev. Lett. 105, 252003 (2010)

13. E. Ruiz Arriola, W. Broniowski, Phys. Rev. D 66, 094016 (2002)

14. W. Broniowski, E. Ruiz Arriola, Phys. Lett. B 773, 385 (2017)

15. W. Broniowski, E. Ruiz Arriola, Phys. Rev. D 97, 034031 (2018)

16. J.R. Gaunt, W.J. Stirling, JHEP 1003, 005 (2010)

17. J. Gaunt, Double Parton Scattering in Proton-Proton Collisions, Ph.D. thesis, Cambridge University, 09 October, 2012 [10.17863/CAM.16589]

18. K. Golec-Biernat, E. Lewandowska, Phys. Rev. D 90, 014032 (2014)

19. M. Diehl, P. Plößl, A. Schäfer, Eur. Phys. J. C 79, 253 (2019)

20. M. Diehl, J.R. Gaunt, D.M. Lang, P. Ploessl, A. Schaefer, Sum Rule Improved Double Parton Distributions in Position Space, arXiv:2001.10428

21. W. Broniowski, E. Ruiz Arriola, Few Body Syst. 55, 381 (2014)

22. W. Broniowski, E. Ruiz Arriola, K. Golec-Biernat, Few Body Syst. 57, 405 (2016)

23. R. Kirschner, Phys. Lett. B 84, 266 (1979)

24. V. Shelest, A. Snigirev, G. Zinovev, Phys. Lett. B 113, 325 (1982)

25. P. Bartalini, J.R. Gaunt, Adv. Ser. Direct. High Energy Phys. 29, 1 (2018)

26. K. Golec-Biernat, E. Lewandowska, M. Serino, Z. Snyder, A.M. Stasto, Phys. Lett. B 750, 559 (2015)

27. E. Ruiz Arriola, W. Broniowski, Phys. Rev. D 67, 074021 (2003) 